\title{
Otimização da Produção de Queijos Usando a Ferramenta Solver do Excel
}

\author{
Optimization of Cheese Production Using the Excel Solver tool
}

\author{
Rosilene Castilho Fernandes Barellaa; Celso Correia de Souza'; José Francisco dos Reis Neto*a; Sidney Maldonado; \\ Wesley Osvaldo Pradella Rodrigues ${ }^{\mathrm{a}}$
}

\begin{abstract}
aUniversidade Anhanguera Uniderp, Programa de Pós-Graduação Stricto Sensu em Produção e Gestão Agroindustrial. MS, Brasil.
*E-mail: jfn@terra.com.br.
\end{abstract}

\begin{abstract}
Resumo
Nos últimos anos, o processo de gestão das empresas tem sido modificado na tentativa de responder mais rapidamente às mudanças ocorridas nas relações empresariais e nos cenários de negócios. A dinâmica é bastante complexa, e requer que os gestores controlem e planejem suas ações na busca pelo sucesso e manutenção de suas empresas no mercado. Este artigo técnico emprega a técnica de Programação Linear (PL) na otimização da produção de queijos em uma empresa de laticínios, em Jaraguari (MS), empregando a ferramenta Solver - Excel para a otimização do lucro. Os dados secundários foram obtidos junto a empresa objeto da pesquisa, a qual apresentava dificuldades na melhoria da sua produção de queijos. Os dados coletados foram organizados para a entrada do Solver, insumos e custos, considerando as restrições produtivas da empresa, para quatro tipos de queijos. Como resultado se demonstrou que o uso da PL na produção de queijos promoveu uma ampliação do lucro à empresa de 30,98\%. Com a realização da análise de sensibilidade, sendo recomendada a fabricação de apenas dois tipos de queijos, considerando os seus custos e preços de venda ao mercado. Este procedimento de tomada de decisão pode ser aplicado em outras atividades de otimização em ambientes da agroindústria e produção sustentável.
\end{abstract}

Palavras-chave: Agronegócio. Derivados do Leite. Pesquisa Operacional. Queijo Coalho.

\begin{abstract}
In recent years, the companies management process has been modified in an attempt to respond more quickly to changes in business relationships and business scenarios. The dynamics are quite complex and require managers to control and plan their actions in the search for the success and maintenance of their companies in the market. This technical article uses the Linear Programming $(P L)$ technique to optimize the production of various types of cheese in a dairy company in Jaraguari (MS), using the Solver - Excel tool to optimize profit. Secondary data were obtained from the research company, which had difficulties in improving its cheese production. The data collected were organized for the entry of the Solver, inputs and costs, considering the company's production restrictions, for four types of cheese. As a result, it is shown that the use of PL in the cheese production promoted an increase in profit of $30.98 \%$ to the company. With the performance of the sensitivity analysis, it was recommended to manufacture only two types of cheese, considering their costs and selling prices to the market. This decision-making procedure can be applied to other optimization activities in agro-industrial and sustainable production environments.
\end{abstract}

Keywords: Agribusiness. Milk Derivatives. Operational Research. Rennet Cheese.

\section{Introdução}

Para facilitar a expansão de mercado e melhor atender os seus consumidores, as empresas procuram alcançar maior eficiência dos seus processos de produção e a pronta resposta às necessidades e desejos do mercado comprador. Ao focar em seus processos produtivos, as modificações e melhorias devem ser ágeis. Segundo destacado por Maximiano (2000), a tomada de decisão é uma tarefa gerencial relevante, desde que se compreenda o desempenho das pessoas e dos processos operacionais. Assim, a empresa consegue aumentar a sua produtividade e diminuir os seus custos, maximizando o lucro.

O empresário do agronegócio, no Brasil, enfrenta problemas na gestão do seu negócio, visto que esse setor da economia é extremamente dinâmico. O agronegócio foi responsável por $23,5 \%$ do Produto Interno Bruto (PIB) do país em 2017, a maior participação em 13 anos, conforme Confederação da Agricultura e Pecuária do Brasil (CNA,
2017).

O Estado de MS tem uma pequena participação no cenário brasileiro na produção de leite. Além da atual baixa produção de leite do Estado, os preços praticados na compra do produto pelas indústrias de laticínios estão muito aquém dos praticados em outros Estados da federação, causando desestímulo aos empresários, inibindo um maior crescimento do setor.

Uma opção aos baixos valores pagos pelo leite em MS é que o próprio produtor de leite promova a industrialização do seu produto, agregando valor ao mesmo, que pode ser vendido no mercado consumidor a um melhor preço. Esse tipo de atividade é pouco praticada no Estado, com pequena quantidade de pequenos laticínios industrializando o leite fornecido pelos pequenos produtores locais. Nesses laticínios são produzidos vários tipos de queijos, leite pasteurizado, bebidas lácteas e outros.

Uma característica do setor industrial dos pequenos laticínios em MS é o gerenciamento da indústria de forma 
intuitiva, sem embasamento científico. Daí, surgiu a seguinte pergunta de pesquisa: a produção desses laticínios poderia ser melhorada com a utilização de gestões mais eficazes? Apesar de as indústrias produzirem satisfatoriamente, remunerando todos os envolvidos no empreendimento, essa remuneração não poderia ser melhorada com a aplicação de ferramentas mais consistentes de gestão? Pensando nisso, este estudo de caso propôs otimizar a produção de um pequeno laticínio na região de Jaraguari (MS), com a aplicação de conceitos de pesquisa operacional.

A pesquisa operacional favorece a tomada de decisão, pois à medida que se tem informações completas em planilhas alimentadas com dados a indústria, o gestor consegue realizar previsões de resultados e estimar riscos de produção, tornando as decisões mais assertivas, propiciando sistemas mais produtivos. Para se obter um valor ótimo do objetivo desejado, as melhorias na distribuição de recursos são realizadas por meio das definições das variáveis de decisão e das restrições de mercado ou de capacidade produtiva (ANDRADE, 2009).

Assim, este estudo teve como objetivo utilizar recursos de programação linear na elaboração de um modelo com a finalidade de estimar a distribuição ótima dos recursos na produção de queijos no laticínio de modo a otimizar seu lucro. Como objetivos específicos se pode destacar: mostrar que a programação linear é uma metodologia matemática robusta capaz de proporcionar melhorias a todos os setores da economia, em especial, para problemas de produção, e contribuir com recomendações de melhores práticas nas ações estratégicas, direcionando a empresa na busca de maior competitividade e efeitos positivos em seu desempenho financeiro.

\section{Desenvolvimento}

\subsection{Metodologia}

O objeto de estudo foi a fábrica de queijos em Jaraguari (MS), que produzia quatro tipos de queijos, cujas quantidades em $\mathrm{kg}$ foram representadas pelos parâmetros: frescal = quantidade produzida, por mês, do queijo tipo minas frescal; padrão = quantidade produzida, por mês, do queijo tipo minas padrão; coalho $=$ quantidade produzida, por mês, do queijo tipo coalho e muçarela $=$ quantidade produzida, por mês, do queijo tipo muçarela, denominadas de variáveis de decisão.

A coleta de dados foi realizada na fábrica de queijos em Jaraguari (MS), no período de maio a julho de 2018, junto aos encarregados da produção e do proprietário. Obteve-se um conjunto de dados primários necessários ao processamento do modelo do problema de PL, com o intento de maximizar o lucro na venda dos produtos, considerando as restrições existentes: mão de obra necessária na produção, quantidade de insumos utilizados para fabricar cada produto e quantidade disponível de cada insumo.

Os dados foram tabulados e organizados em planilhas do Microsoft Excel, para a utilização da ferramenta Solver-Excel
(FONSECA et al, 2017; GOMES JUNIOR; SOUZA, 2014;

\section{LAPPONI, 2008; LOURENZANI; LOURENZANI, 2006).}

Com o modelo matemático definido, o Solver disponibiliza dois relatórios, nos quais e é possível identificar a solução ótima - através do relatório de resposta, e avaliar os reflexos sobre eventuais alterações nos dados e condições do problema - através do relatório de sensibilidade.

\subsection{Discussão}

A fábrica de queijos, fiscalizada pelo Serviço de Inspeção Estadual de MS (SIE), da Agência Estadual de Defesa Sanitária Animal e Vegetal (IAGRO), produzia à época quatro tipos de queijos com suas respectivas produções mensais: minas frescal; minas padrão, coalho e muçarela, com produções mensais médias de 1.436,89 kg; 954,64 kg; 438,55 $\mathrm{kg}$ e $1.761,67 \mathrm{~kg}$, respectivamente, e utiliza cinco insumos: leite; coagulante; ácido lático; cloreto de cálcio a $40 \%$ e sal.

Na rotina de produção, o fabricante usa uma formulação com quantidades fixas de insumos para produção dos seus queijos, variando assim, o rendimento de cada formulação: $69,58,55$ e $120 \mathrm{~kg}$ de queijos tipos minas frescal, minas padrão, coalho e muçarela, respectivamente. O volume de leite comprado mensalmente era de $53.500 \mathrm{~L}$, utilizando-se 2.500 L em cada produção. Eram produzidas aproximadamente 21 bateladas ao mês (Quadro 1).

Quadro 1 - Descrição das quantidades específicas de cada insumo e rendimento dos queijos por batelada

\begin{tabular}{|c|c|c|c|c|c|}
\hline \multirow[b]{2}{*}{ Insumos } & \multicolumn{4}{|c|}{ Produtos } & \multirow[b]{2}{*}{ Total } \\
\hline & $\begin{array}{c}\text { Queijo } \\
\text { Minas } \\
\text { Frescal }\end{array}$ & $\begin{array}{c}\text { Queijo } \\
\text { Minas } \\
\text { Padrão }\end{array}$ & $\begin{array}{l}\text { Queijo } \\
\text { Coalho }\end{array}$ & $\begin{array}{c}\text { Queijo } \\
\text { Muçarela }\end{array}$ & \\
\hline Leite (L) & 500 & 500 & 500 & 1000 & 2500 \\
\hline $\begin{array}{l}\text { Coagulante } \\
(\mathrm{mL})\end{array}$ & 20 & 20 & 20 & 42 & 102 \\
\hline $\begin{array}{l}\text { Cloreto } \\
\text { de Cálcio } \\
(\mathrm{mL})\end{array}$ & 130 & 130 & 130 & 240 & 630 \\
\hline \begin{tabular}{|l} 
Ácido \\
Lático \\
$(\mathrm{mL})$ \\
\end{tabular} & 160 & 160 & 0 & 0 & 320 \\
\hline Sal (kg) & 3 & 3 & 4 & 8 & 18 \\
\hline $\begin{array}{l}\text { Produção } \\
\mathrm{kg} / \text { batelada }\end{array}$ & 69 & 58 & 55 & 120 & 302 \\
\hline
\end{tabular}

Fonte: Dados da pesquisa.

Os valores mensais dos custos de produção foram obtidos a partir das notas fiscais apresentadas e para cada tipo de queijo foram calculados os custos com o leite, coagulante, cloreto de cálcio, ácido lático, sal e horas/homem, incluindo custos de embalagens e rótulos, estimados em reais (Quadro 2). 
Quadro 2 - Descrição dos custos em reais para produção de 1 kg de cada tipo de queijo

\begin{tabular}{|l|c|c|c|c|}
\hline \multirow{2}{*}{ Despesas } & \multicolumn{4}{|c|}{ Produtos } \\
\cline { 2 - 5 } & $\begin{array}{c}\text { Queijo } \\
\text { Minas } \\
\text { Frescal }\end{array}$ & $\begin{array}{c}\text { Queijo } \\
\text { Minas } \\
\text { Padrão }\end{array}$ & $\begin{array}{c}\text { Queijo } \\
\text { Coalho }\end{array}$ & $\begin{array}{c}\text { Queijo } \\
\text { Muçarela }\end{array}$ \\
\hline Leite & 8.70 & 10.34 & 10.91 & 12.00 \\
\hline Salários & 5.53 & 6.58 & 6.94 & 3.82 \\
\hline Telefone & 0.00 & 0.04 & 0.05 & 0.03 \\
\hline $\begin{array}{l}\text { Energia } \\
\text { Elétrica }\end{array}$ & 0.83 & 0.99 & 1.05 & 0.58 \\
\hline Transportes & 1.32 & 1.65 & 1.74 & 0.95 \\
\hline Outras & 0.01 & 0.01 & 0.01 & 0.01 \\
\hline Coagulante & 0.07 & 0.09 & 0.09 & 0.09 \\
\hline $\begin{array}{l}\text { Cloreto de } \\
\text { Cálcio }\end{array}$ & 0.02 & 0.02 & 0.02 & 0.02 \\
\hline $\begin{array}{l}\text { Ácido } \\
\text { Lático }\end{array}$ & 0.08 & 0.10 & 0.00 & 0.00 \\
\hline Sal & 0.03 & 0.04 & 0.06 & 0.06 \\
\hline Embalagem & 4.32 & 4.32 & 1.50 & 0.67 \\
\hline Rótulo & 0.10 & 0.10 & 0.14 & 0.05 \\
\hline Custo & $\mathbf{2 1 . 0 1}$ & $\mathbf{2 4 . 2 8}$ & $\mathbf{2 2 . 4 9}$ & $\mathbf{1 8 . 2 7}$ \\
\hline
\end{tabular}

Fonte: Dados da pesquisa.

Os custos fixos mensais foram divididos entre salários operacional e administrativo, despesas com telefone, energia elétrica, transporte e outras menos expressivas. Foram incluídas também despesas com equipamentos de proteção individual para a manipulação, como: luvas, toucas e máscaras, também descritos no Quadro 2.

Os valores de venda por $\mathrm{kg}$ dos produtos são $\mathrm{R} \$ 26,90$ para os queijos minas frescal e minas padrão, $\mathrm{R} \$ 23,90$ para o queijo coalho e $\mathrm{R} \$ 20,00$ para a muçarela. A partir das informações coletadas se considerou que o lucro de produção da fábrica de queijos (L) é igual a receita (R) menos custos (C), $(\mathrm{L}=\mathrm{R}-\mathrm{C})$. $\mathrm{O}$ custo total foi calculado e dividido por $\mathrm{kg}$ de queijo produzido, gerando um modelo matemático da função lucro L a ser maximizada, denominada de função objetivo.

Para este estudo foram realizadas duas simulações do modelo matemático de PL, sendo considerado na simulação 1: o coagulante, o ácido lático, sal e leite como restrições e nenhuma restrição de mercado e, na simulação 2: mantendo as restrições com exigências mínimas de mercado de $40 \%$ da produção. Na simulação 1, o modelo de PL, composto da função objetivo L e o conjunto de restrições é dado pelo modelo, equação (1).

Máx.L $=5,89$ frescal $+2,62$ padrao $+1,41$ coalho $+1,73$ muçarela

Sujeito às restrições, equação (2).

Máx.Z=5,892 x_1+2,622 x_2+1,407 x_3+1,731x_4

\subsubsection{Solução pelo Solver}

O primeiro passo consistiu em criar uma planilha de cálculo no Excel, explicitando as células nas quais foram colocados os valores das variáveis de decisão, que iniciaram com valores zeros; os coeficientes da função objetivo; os coeficientes da matriz das restrições e as constantes que constituem o lado direito das restrições (LD). O Quadro 3 apresenta a planilha de cálculo descrita anteriormente.

Quadro 3 - Planilha de cálculo no Excel para a implementação do Solver na otimização de laticínio, as variáveis de decisão, a fórmula que relaciona estes coeficientes com as variáveis de decisão (FO), os lados esquerdo e direito das restrições (LE) e (LD), respectivamente

\begin{tabular}{|c|c|c|c|c|}
\hline \multicolumn{5}{|c|}{ Otimização de Laticínio } \\
\hline \multicolumn{5}{|c|}{ Variáveis de Decisão } \\
\hline Frescal & padrão & coalho & muçarela & \\
\hline 0 & 0 & 0 & 0 & \\
\hline \multicolumn{5}{|c|}{ Função Objetivo (FO) } \\
\hline Frescal & padrão & coalho & muçarela & \\
\hline 5,892 & 2,622 & 1,407 & 1,731 & Restrições \\
\hline \multicolumn{5}{|c|}{} \\
\hline \multicolumn{5}{|c|}{ Lado Esquerdo (LE) } \\
\hline 0,290 & 0,345 & 0,364 & 0,420 & 102 \\
\hline 1,884 & 2,241 & 2,364 & 2,400 & 630 \\
\hline 0,043 & 0,052 & 0,073 & 0,080 & 18 \\
\hline 7,246 & 8,621 & 9,091 & 10,000 & 2500 \\
\hline 2,319 & 2,759 & 0,000 & 0,000 & 320 \\
\hline
\end{tabular}

Fonte: Dados da pesquisa.

O próximo passo foi inserir na planilha de cálculo criada no Excel a fórmula que relaciona os coeficientes da função objetivo (FO) com as variáveis de decisão e a fórmula que relaciona os coeficientes do lado esquerdo das restrições (LE) com as variáveis de decisão. A Tabela 4 apresenta as fórmulas no Excel, sem considerar as igualdades antes dessas, para a explicitação das expressões, sem que os cálculos sejam realizados. À direita da tabela 4 foram consideradas as correspondentes células com as respectivas igualdades, para explicitar os resultados dos cálculos.

Quadro 4 - Planilha de cálculo do Excel, mostrando as equações inseridas, sem colocar o símbolo $(=)$ antes das mesmas e com os sinais de igualdade explicitando os valores dos cálculos realizados

\begin{tabular}{|c|c|c|c|}
\hline FO & & FO & \\
\hline $\mathrm{J} 12 * \mathrm{~J} 8+\mathrm{K} 12 * \mathrm{~K} 8+\mathrm{L} 12 * \mathrm{~L} 8+\mathrm{M} 12 * \mathrm{M} 8$ & & 0 & \\
\hline LE & LD & LE & LD \\
\hline
\end{tabular}

\begin{tabular}{|l|l|l|l|l|}
\hline $\mathrm{J} 16 * \$ \mathrm{~A} \$ 8+\mathrm{K} 16 * \$ \mathrm{~B} \$ 8+\mathrm{L} 16 * \$ \mathrm{C} \$ 8+\mathrm{M} 16 * \$ \mathrm{D} \$ 8$ & 102 & 0 & 102
\end{tabular} \begin{tabular}{|l|l|l|l|}
\hline $\mathrm{J} 17 * \$ \mathrm{~A} \$ 8+\mathrm{K} 17 * \$ \mathrm{~B} \$ 8+\mathrm{L} 17 * \$ \mathrm{C} \$ 8+\mathrm{M} 17 *$ \$D $\$ 830$ & 0 & 630
\end{tabular} \begin{tabular}{|l|l|c|c|}
\hline $\mathrm{J} 18 * \$ \mathrm{~A} \$ 8+\mathrm{K} 18 * \$ \mathrm{~B} \$ 8+\mathrm{L} 18 * \$ \mathrm{C} \$ 8+\mathrm{M} 18 * \$ \mathrm{D} \$ 8$ & 18 & 0 & 18 \\
\hline
\end{tabular}

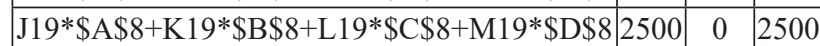
\begin{tabular}{|l|l|l|l|l|}
\hline $\mathrm{J} 20 * \$ \mathrm{~A} \$ 8+\mathrm{K} 20 * \$ \mathrm{~B} \$ 8+\mathrm{L} 20 * \$ \mathrm{C} \$ 8+\mathrm{M} 20 * \$ \mathrm{D} \$ 8$ & 320 & 0 & 320 \\
\hline
\end{tabular}

Fonte: dados da pesquisa.

Para a resolução desse problema, basta entrar na ferramenta Solver do Excel. Esse "suplemento" do Excel não é instalado quando da instalação do Excel em modo típico ou mínimo. 
Apenas é instalada em modo completo ou personalizando a instalação.

Clicando no menu "Dados" e, depois, em "Solver", surge a seguinte caixa de diálogo, Figura 1.

Figura 1 - Caixa de diálogo da ferramenta Solver do Excel para a introdução dos dados do problema

Parâmetros do Solver

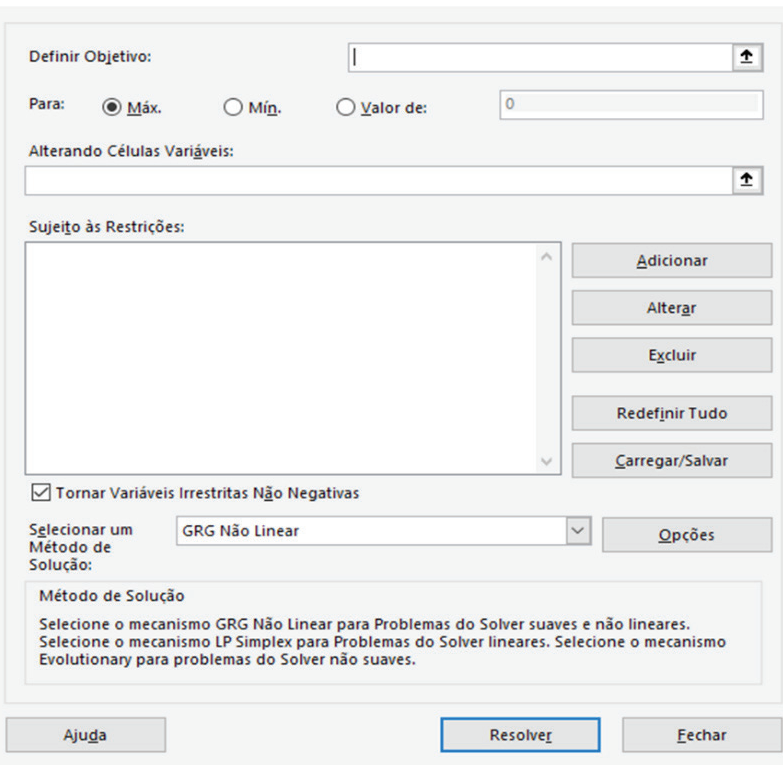

Fonte: Adaptado de Lapponi (2008).

A introdução dos dados do problema é realizada seguindo os seguintes passos:

- Como célula de destino se indica a localização da função objetivo;

- Seguidamente indica-se o tipo da otimização;

- No campo "Células variáveis" se indica a referência das células correspondentes às variáveis de decisão.

- As restrições serão introduzidas através do botão "Adicionar" que abre a caixa de diálogo da Figura 2.

Figura 2 - Caixa de diálogo do Solver para a introdução das restrições do problema

Adicionar Restrição

\begin{tabular}{|c|c|c|c|c|}
\hline \multicolumn{4}{|c|}{ Referência de Célula: } & Restrição: \\
\hline | & $\underline{\boldsymbol{1}}$ & $<=$ & $\checkmark$ & $\underline{\imath}$ \\
\hline$\underline{\mathrm{O} K}$ & & Adi & & Cancelar \\
\hline
\end{tabular}

Fonte: Adaptado de Lapponi (2008).

Na caixa "Referência da Célula" se introduz a referência da célula com o lado esquerdo da restrição:

- Na caixa "Restrição" se introduz a referência da célula com o lado direito da restrição. No menu do meio se seleciona o tipo de restrição: $\geq, \leq$ ou $=$;

- Também, é aqui que se declaram as variáveis como inteiras ou binárias, que não é o caso deste exemplo;

- Introduzidos os dados referentes a uma restrição se pressiona o botão "Adicionar";

- Depois de adicionadas todas as restrições, terminar pressionando o botão "Cancelar".

É possível introduzir mais do que uma restrição de uma vez. Se as restrições tiverem o mesmo sentido de desigualdade se usa a forma: $\$ G \$ 16: \$ G \$ 20<=\$ H \$ 16: \$ H \$ 20$.

Executando essa tarefa para todas as restrições obtém-se a Figura 3.

Figura 3 - Caixa de diálogo da ferramenta Solver do Excel com todos os dados do problema já inseridos

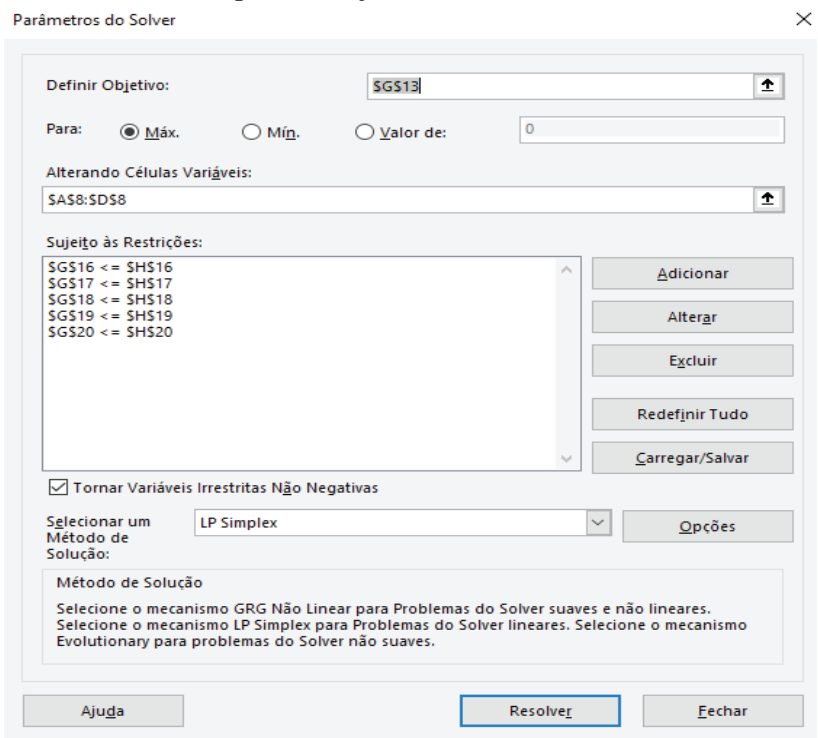

Fonte: Adaptado de Lapponi (2008).

Na Figura 3, na caixa "Selecionar o método de solução", coloque "LP Simplex" para o presente caso. A caixa "Opções" permite resolver outros modelos, como as programações "Não-linear" e "Evolucionária". Clicando em "Resolver" aparecerá uma caixa de duas caixas de diálogos possíveis: i) o Solver não encontrou solução factível e; ii) o Solver encontrou a solução. Nesse último caso aparecerá a Figura 4, para se fazer a seleção de relatórios de saídas: Resposta, Sensibilidade e Limites.

Figura 4 - Caixa de diálogo do Solver com as opções de relatórios de saídas

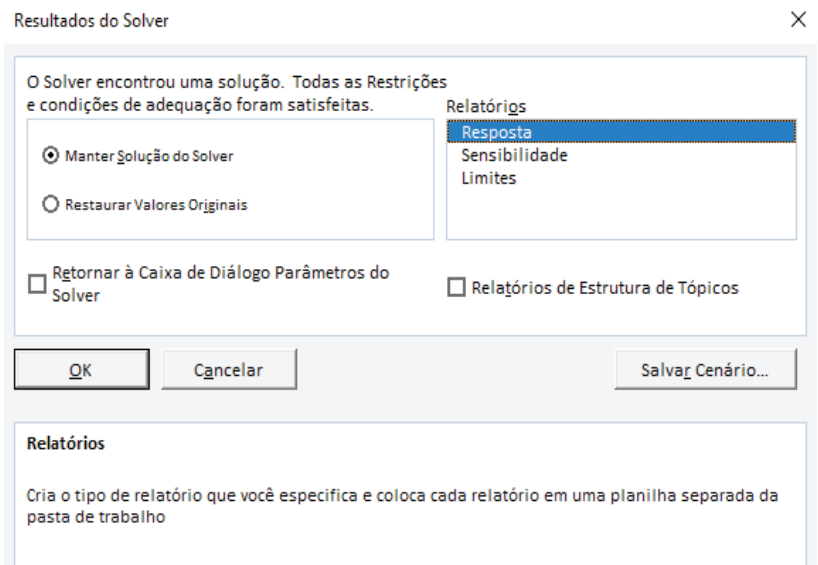

Fonte: Adaptado de Lapponi (2008).

Como só interessa a resposta ao problema, foi selecionada a opção "Resposta”, surgindo o relatório do Quadro 5. 
Quadro 5 - Apresentação dos resultados da solução do problema utilizando a opção "Resposta"

\begin{tabular}{|c|c|c|c|c|c|}
\hline Célula & Nome & Valor Original & Valor Final & & \\
\hline$\$ G \$ 13$ & FO & 0 & 1068,4976 & & \\
\hline Célula & Nome & Valor Original & Valor Final & Número Inteiro & \\
\hline$\$ A \$ 8$ & frescal & 0 & 137,9905 & Conting. & \\
\hline$\$ B \$ 8$ & coalho & 0 & 0 & Conting. & \\
\hline$\$ C \$ 8$ & padrão & 0 & 0 & Conting. & \\
\hline$\$ D \$ 8$ & muçarela & 0 & 147,5780 & Conting. & \\
\hline Célula & Nome & Valor da Célula & Fórmula & Status & Margem de Atraso \\
\hline$\$ G \$ 16$ & LE & 102 & $\$ G \$ 16<=\$ H \$ 16$ & Associação & 0 \\
\hline$\$ \mathrm{G} \$ 17$ & LE & 614,1613 & $\$ G \$ 17<=\$ H \$ 17$ & Não-associação & 15,8387 \\
\hline$\$ G \$ 18$ & $\mathrm{LE}$ & 17,7398 & $\$ G \$ 18<=\$ H \$ 18$ & Não-associação & 0,2602 \\
\hline$\$ G \$ 19$ & LE & 2475,6590 & $\$ G \$ 19<=\$ H \$ 19$ & Não-associação & 24,3410 \\
\hline$\$ G \$ 20$ & LE & 320 & $\$ G \$ 20<=\$ H \$ 20$ & Associação & 0 \\
\hline
\end{tabular}

Fonte: Adaptado de Lapponi (2008).

Do Quadro 5 se conclui que a solução ótima encontrada pelo Solver, indicando ser esse o mix de produtos que gera a melhor margem de contribuição, foi a produção de $138 \mathrm{~kg}$ de queijo Minas Frescal; $148 \mathrm{~kg}$ de queijo Muçarela e a não produção dos tipos de queijos Minas Padrão e Coalho, com lucro de $\mathrm{R} \$ 1.068,50$ por batelada e um lucro de $\mathrm{R} \$ 22.438,50$ mensal (não está na solução do Solver este valor). O relatório de restrições indica não haver sobras dos insumos Coagulante e Ácido Lático, com sobra de 24,34 L de Leite, $630 \mathrm{~mL}$ de Cloreto de Cálcio e $0,26 \mathrm{~kg}$ de Sal.

Pela solução obtida, pode ser feita a comparação dos ganhos, que advêm da ampliação da capacidade restritiva da empresa, com os eventuais custos envolvidos. Esse representa a quantidade pela qual a função objetivo é alterada, dado um acréscimo de uma unidade na constante da restrição, assumindo que todos os outros coeficientes e constantes das restrições se conservam inalterados. A interpretação econômica seria: até quanto se está disposto a pagar por uma unidade adicional de um recurso para aumentar a produção do respectivo produto (LACHTERMACHE, 2012).

Com a análise de sensibilidade foram investigados os efeitos que determinadas alterações realizadas nos parâmetros do PL causariam na solução ótima (BELFIORE; FÁVERO, 2013), revelando as margens de contribuição unitária que cada produto pode variar em um intervalo. O Quadro apresenta o relatório de sensibilidade obtida pelo Solver, quando se escolhe a opção sensibilidade na Figura 4.

Figura 6 - Relatório de sensibilidade apresentado pela ferramenta Solver do Excel

\begin{tabular}{|c|c|c|c|c|c|c|}
\hline \multicolumn{7}{|c|}{ Células Variáveis } \\
\hline & & Final & Reduzido & Objetivo & Permitido & Permitido \\
\hline Célula & Nome & Valor & Custo & Coeficiente & Aumentar & Reduzir \\
\hline$\$ A \$ 8$ & frescal & 137,9905 & 0 & 5,8920 & $1 \mathrm{E}+30$ & 3,6881 \\
\hline$\$ B \$ 8$ & coalho & 0 & $-4,3878$ & 2,6220 & 4,3878 & $1 \mathrm{E}+30$ \\
\hline$\$ C \$ 8$ & padrão & 0 & $-0,0932$ & 1,4070 & 0,0932 & $1 \mathrm{E}+30$ \\
\hline \$D\$8 & muçarela & 147,5780 & 0 & 1,7310 & 6,8022 & 0,1075 \\
\hline \multicolumn{7}{|l|}{ Restrições } \\
\hline & & Final & Sombra & Restrição & Permitido & Permitido \\
\hline Célula & Nome & Valor & Preço & $\mathbf{L E}$ & Aumentar & Reduzir \\
\hline$\$ G \$ 16$ & $\mathrm{LE}$ & 102 & 4,1214 & 102 & 1,0223 & 61,9828 \\
\hline$\$ G \$ 17$ & LE & 614,1613 & 0 & 630 & $1 \mathrm{E}+30$ & 15,8387 \\
\hline \$G\$18 & LE & 17,7398 & 0 & 18 & $1 \mathrm{E}+30$ & 0,2602 \\
\hline$\$ G \$ 19$ & LE & 2475,6590 & 0 & 2500 & $1 \mathrm{E}+30$ & 24,3410 \\
\hline$\$ G \$ 20$ & LE & 320 & 2,0253 & 320 & 161,9081 & 49,2996 \\
\hline
\end{tabular}

Fonte: Adaptado de Lapponi (2008).

\subsubsection{Preço Sombra}

Existirá preço-sombra só para restrições sem folga ou sobra zero. O preço-sombra zero indica a faixa de aumentos ou decréscimos permissíveis sem que haja mudanças no resultado ótimo encontrado para a função-objetivo. O preçosombra indica quanto o valor da função-objetivo irá variar, dadas possíveis mudanças na disponibilidade da restrição. No entanto, esse não diz quais valores as variáveis de decisão terão que assumir para atingir o novo valor da função objetivo. Para tal é necessário reprocessar o solver (refazer).

$\mathrm{Na}$ situação estudada, poderia ser pago um extra de R\$ 2,03 para cada unidade adicional de Ácido Lático e ser pago uma extra de $\mathrm{R} \$ 4,12$ para cada unidade adicional de Coagulante, para se manter a Margem de Contribuição Total original.

O Laticínio, no momento da pesquisa, produzia $69,58,55$ 
e $100 \mathrm{~kg}$ dos respectivos queijos minas frescal, minas padrão, coalho e muçarela, com valores de venda por $\mathrm{kg}$ de $\mathrm{R} \$ 26,90$ para os queijos minas frescal e minas padrão, $\mathrm{R} \$ 23,90$ para o queijo coalho e $\mathrm{R} \$ 20,00$ para a muçarela, com um lucro $\mathrm{L}=\mathrm{R} \$ 809,11$. Aplicando a ferramenta PL de gestão o seu lucro passaria a R\$1.068,50, um valor $30,98 \%$ acima do que o laticínio vinha lucrando.

Em uma nova simulação, foi mantida a função objetiva e o modelo de PL, composto da função objetivo, com o conjunto de restrições dado pelo modelo da equação (4).

Máx.Z=5,892frescal+2,622padrão+1,407coalho+1,731m uçarela

Sujeito a seguintes restrições, equação (5).

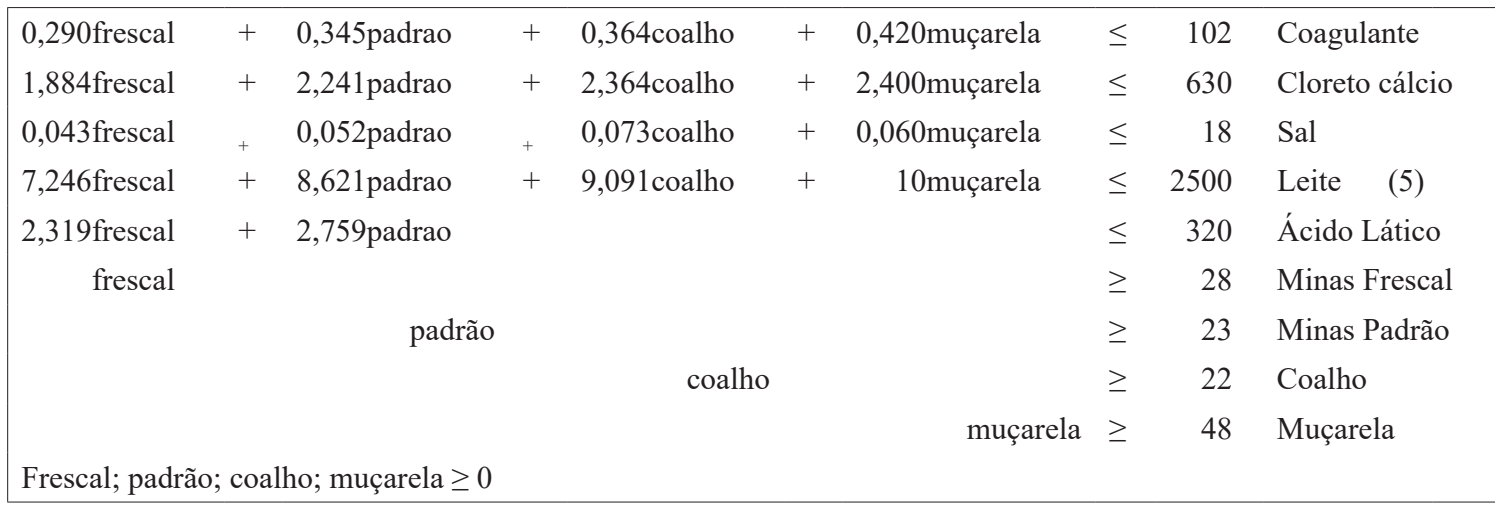

Acrescentando ao modelo restrições de mercado, isto é, exigências mínimas de mercado de $40 \%$ da produção dos quatro tipos de queijo, a nova solução ótima encontrada pelo Solver indicava ser esse o mix de produtos que geraria a melhor margem de contribuição: $111 \mathrm{~kg}$ de minas frescal e 23 $\mathrm{kg}$ de minas padrão, $22 \mathrm{~kg}$ de coalho e $129 \mathrm{~kg}$ de muçarela, com lucro de R\$958,56 por batelada e um lucro de $\mathrm{R} \$$ 20.129,76 mensal.

Ficou comprovado que os queijos minas padrão e coalho têm problemas de mercado, pois só se deve produzir as quantidades mínimas para o atendimento do mercado. Análises semelhantes poderiam ser feitas sobre o relatório de sensibilidade, que não foi rodado nessa simulação. Observe que, mesmo impondo as restrições de mercado em 40\%, aplicando a ferramenta PL, o lucro foi de R \$ 958,56, que foi de $18,47 \%$ acima do que o laticínio vinha lucrando atualmente.

\section{Conclusão}

A utilização da PL, implementada com a ferramenta Solver do Excel, na produção de queijos, particularmente no Laticínio de Jaraguari, MS, se mostrou muito eficiente, propiciando ao proprietário e/ou gestores do Laticínio informações úteis para as tomadas de decisão, quando da produção de queijos com os menores custos possíveis, o que favorece a concorrência em um mercado altamente competitivo como esse dos laticínios.

Observou-se nos resultados que os lucros desse Laticínio de Jaraguari poderiam alcançar uma elevação do lucro de $30,98 \%$ se passasse a utilizar as análises realizadas. Com a realização da análise de sensibilidade, novas possibilidades são incorporadas à solução do problema, se vale a pena investir na compra de insumos para aumentar a produção, visando um maior lucro. Esse procedimento de tomada de decisão pode ser aplicado em outras atividades de otimização em ambientes da agroindústria e produção sustentável.

\section{Referências}

ANDRADE, E.L. Introdução à pesquisa operacional: métodos e modelos para análise de decisões. Rio de Janeiro: LTC, 2009.

BELFIORE, P.; FÁVERO, L. Pesquisa operacional para cursos de Engenharia. Rio de Janeiro: Elsevier, 2013.

CNA-Brasil. Confederação da Agricultura e Pecuária do Brasil. O agronegócio cresceu. 2017. Disponível em: www.cnabrasil. org.br/pib. Acesso em: 26 maio 2020.

FIESP - Outlook Fiesp 2023: Projeções para o Agronegócio Brasileiro / Federação das Indústrias do Estado de São Paulo. São Paulo: FIESP, 2013.

FONSECA, G.S.; VINHAL, N.M.; MOURA JR, J.R.V. Utilizando o solver para resolver problemas de programação da produção com o método de programação inteira. In: PITUBA, J.J.C.; STOPPA, M.H.. (Org.). Tecnologias em pesquisa: engenharias. São Paulo: Blucher, 2017. p.273-282.

GOMES JÚNIOR, A.C.; SOUZA, M.J.F. Solver (Excel): manual de referência. 2014. Disponível em: http://www.decom.ufop.br/ prof/marcone/Disciplinas/OtimizacaoCombinatoria/solver_p. pdf, Acesso em: 20 set. 2020.

LACHTERMACHER, G. Pesquisa operacional na tomada de decisões. São Paulo: Pearson Prentice Hall, 2012.

LAPPONI, J.C. Modelagem Financeira com Excel e VBA. São Paulo: Campus, 2008.

LOURENZANI, W. L.; LOURENZANI, A. E. B. S. Potencialidades do agronegócio brasileiro do amendoim. Soc. Bras. Economia Soc. Rural, n.44, 2006.

MAXIMIANO, A. C. Introdução à Administração. São Paulo: Atlas, 2000.

ZOCCAL, R. A força do agro e do leite no Brasil. São Paulo: Balde Branco, 2017. 